\title{
Repräsentation in der (Internationalen) Politischen Theorie - eine feministische Kritik und ein Aufruf zum Dialog
}

\author{
Leonie Holthaus $\cdot$ Henrike Knappe $\cdot$ Marina Martinez Mateo
}

Eingegangen: 16. Februar 2021 / Überarbeitet: 14. Januar 2022 / Angenommen: 14. Januar 2022 / Online publiziert: 21. Februar 2022

(C) Der/die Autor(en) 2022

Zusammenfassung Wer darf für wen jenseits des Staates sprechen, und welche Identitäten werden durch dieses Sprechen erst angeboten und erzeugt? Auch wenn die theoretische wie praktische Relevanz dieser Fragen klar zu ersehen ist, bergen sie eine Reihe von Herausforderungen. Wir möchten in einer Replik auf eine 2020 erschienene Debatte Verweise auf sehr relevante wie schwierige Fragen zu transnationaler Repräsentation mit der These verbinden, dass die feministische Beschäftigung mit transnationaler Repräsentation exemplarisch Ansätze zur Verbindung von normativer und empirischer Forschung zur Verfügung stellt. Bedauerlicherweise wurde die feministische Repräsentationstheorie und die Inspiration, die von ihr ausgeht, in der Debatte nicht beachtet. Über eine fokussierte Besprechung der Beiträge der Internationalen Beziehungen und der Politischen Theorie zeigen wir, dass feministische Interventionen im Spannungsfeld zwischen Theorie und Praxis stetige Kritik an aufkommenden Ungleichheiten und Identitätskonstruktionen leisten. Darin schließlich tragen sie zu einer Verunsicherung von theoretischen Vorannahmen bei, die für eine empirisch informierte Politische Theorie entscheidend ist.

Schlüsselwörter Repräsentation · Globale Demokratie · Feminismus · Theoriedialog $\cdot$ Ethnographische Methoden

\footnotetext{
Leonie Holthaus $(\bowtie)$

Technische Universität Darmstadt, Darmstadt, Deutschland

E-Mail: holthaus@pg.tu-darmstadt.de

Henrike Knappe

Institut für transformative Nachhaltigkeitsforschung (IASS), Potsdam, Deutschland

Marina Martinez Mateo

Kunstakademie München, München, Deutschland
} 


\title{
Representation in (International) Political Theory-A Feminist Critique and a Call for Dialogue
}

\begin{abstract}
Who is allowed to speak for whom beyond the state, and which identities are offered and created by this act of speaking? The theoretical and practical relevance of these questions is clear, but the questions imply a number of challenges. In a response to a debate published in 2020, we zoom in on questions of transnational representation before we argue that feminist studies of transnational representation provide critical examples for how to address transnational representation through approaches that combine normative and empirical research. Feminist representation theory and the inspiration that can be derived from it have been left out of the debate. Hence, and through a focused discussion of the contributions of international relations and political theory, we show that feminist interventions bridge theory and practice and provide constant critiques of emerging inequalities and constructions of identity. Feminist representation theory enriches reflections on theoretical presuppositions and moves political theories that are informed by empirical analysis.
\end{abstract}

Keywords Representation - Global democracy · Feminism • Theoretical dialogue · Ethnographic methods

\section{Einleitung}

Dieser Beitrag verbindet eine Replik auf die 2020 veröffentlichte Debatte zum Stellenwert der Politischen Theorie (PT) und Ideengeschichte im Wissenschaftssystem (Niesen et al. 2020) mit Verweisen auf hochrelevante wie schwierige Fragen zu transnationaler Repräsentation. Wir vertreten die These, dass die feministische Beschäftigung mit transnationaler Repräsentation exemplarisch Ansätze zur Verbindung von normativer und empirischer Forschung zur Verfügung stellt und zugleich zeigt, wie komplex und problembehaftet solche Verbindungen sind.

In der Debatte wurde die (Selbst-)Identifikation PT mit normativen Zugängen gelobt wie auch kritisiert. Übereinstimmung herrscht demzufolge hinsichtlich der Relevanz normativer Fragen, wie sie in der PT gestellt werden. Gleichzeitig wurde direkte Kritik an der Aufstellung der PT geübt, da sie traditionell normative Fragen auf Kosten von bzw. ohne jedwede empirische Betrachtung anstelle. Dies begünstige einen spezifischen akademischen Habitus. Wie Michael Zürn schreibt, zeichnet er sich durch ein Selbstbewusstsein als verpasste (intellektuelle) Avantgarde, die sich mit überlegenden normativen Fragen beschäftigt, aus, à la „Daten sammeln und auswerten dürfen die anderen, wir übernehmen das Denken" (Niesen et al. 2020, S. 755). Diese Beanspruchung (überlegender) Normativität ist teils auch in Diagnosen zum Verhältnis von PT und den Internationalen Beziehungen (IB) zu finden (Niesen 2010, S. 275). Gerade im deutschen Kontext wird häufig davon ausgegangen, dass die IB bei normativen und demokratischen Fragen auf die PT angewiesen sei. Schließlich verfüge sie nicht über eigene normative Ressourcen und Reflexionskapazitäten. Mittlerweise erzeugt diese Position allerdings auch Gegenrede (Deitelhoff und Daase 2015). 
Die Positionierungen und genannten Überlegungen verweisen auf eine hochrelevante Kontroverse. Dennoch erkennen wir in der Darstellung der PT Vereinfachungen, auf die wir hier reagieren möchten. Die vorgenommenen Darstellungen gehen kaum auf interne Differenzen geschweige denn interne Hierarchien innerhalb des Feldes der PT ein. Es erfolgt stattdessen eine schnelle Identifikation mit Theorie von normativen Ansätzen. Die von dem empirischen Problem der Geschlechterungleichheit angetriebene feministische Forschung findet kaum Beachtung. Dabei ist die feministische Forschung weniger als eigene (Sub-)Disziplin und vielmehr als disziplinübergreifende Reflexion von Machtverhältnissen zu verstehen.

Um Probleme und Potenzial der empirischen Öffnung der PT konkret zu diskutieren, widmet sich der Beitrag dem Thema der transnationalen Repräsentation aus feministischer Perspektive. Die Wahl ist dem weiten Interesse an transnationaler Demokratie geschuldet, mit dem Fragen um Repräsentation jenseits des Staates an Relevanz gewinnen. Zudem wird die schwierige wie fruchtbare Verbindung von normativen und empirischen Fragen hier besonders deutlich. Schließlich manifestiert sich transnationale Repräsentation primär in den Praktiken transnationaler zivilgesellschaftlicher Akteure, wie auch transnationaler feministischer Bewegungen, und eher selten in Imitationen nationalstaatlicher, parlamentarischer Repräsentation. Dies impliziert, dass demokratische Konzepte, ausgehend von politischen Praktiken und empirischen Fragen, geöffnet und neu gedacht werden müssen. Diese Praktiken sind äußerst divers und mit einer Vielzahl von epistemischen wie materiellen Ungleichheiten verbunden.

$\mathrm{Zu}$ diesem Zweck ist der Beitrag wie folgt aufgebaut: Wir werden erst das staatszentrische Repräsentationsverständnis aufrufen und problematisieren, bevor wir auf die disziplinspezifischen Diskurse und Innovationen in der PT und IB eingehen. Schließlich verweisen wir auf die feministische Repräsentationstheorie, in der normative und empirische Zugänge bereits miteinander verwoben werden. Die Repräsentationsforschung sowohl in der PT als auch in der IB kann, so unser Argument, durchaus inspiriert werden durch die Ansätze in der feministischen Repräsentationstheorie.

\section{Fragen transnationaler Repräsentation}

In der PT ist die staatszentrische Tradition für das Repräsentationsverständnis nach wie vor dominant: staatliche Institutionen stehen hier im Zentrum der Politik. Einerseits wird diese Tradition in systematischen Analysen vorausgesetzt. So wird im Anschluss an Hannah Pitkin (1972) auch heute weiter zwischen etwa formeller, deskriptiver und substanzieller Repräsentation unterschieden (Dovi 2006). Andererseits arbeiten ideengeschichtliche Ansätze die Bedeutung von Repräsentation in der Herausbildung staatlicher Souveränität heraus, sodass hier die enge Verknüpfung von Repräsentation und Souveränität stabilisiert wird (Martinez Mateo 2018; Duso 2006; Hofmann 2003). Implizit oder explizit bleibt der Repräsentationsbegriff insofern primär auf staatliche Institutionen (etwa Parlamente oder Regierungen) und formalisierte Verfahren (etwa Wahlen) bezogen. Zugleich ist damit vorausgesetzt, dass es ein definierbares Kollektiv gibt (Staatsvolk oder Demos), das überhaupt 
durch diese Institutionen repräsentiert werden soll und entsprechend etwa berechtigt ist $\mathrm{zu}$ wählen.

Dass diese Perspektive nicht den vielfältigen Demokratieansprüchen im transnationalen Kontext gerecht wird, ist innerhalb der PT anerkannt (vgl. beispielhaft Urbinati und Warren 2008), und neuere Ansätze zur Etablierung eines breiteren und dynamischen Verständnisses von Repräsentation werden prinzipiell begrüßt. Doch Kritiken wie etwa Schmitters scharfe Zurückweisung eines dezidiert deterritorialisierten Repräsentationsverständnisses zeigen, dass radikale Neuerungen weiterhin mit Gegenwehr zu rechnen haben (Macdonald 2008; Schmitter 2009). Nichtsdestotrotz sind deutliche Verschiebungen in aktuellen Theorien des Repräsentationsverständnisses nachzuzeichnen, durch die sich die PT (zumindest potenziell) als anschlussfähig für die IB erweist. Zwei Merkmale dieser Verschiebungen möchten wir im Folgenden hervorheben.

Erstens wird die Annahme, dass es einen vordefinierten Demos geben muss, dessen Willen durch entsprechende (möglichst akkurate) Verfahren möglichst gut abgebildet werden soll, in neueren Repräsentationsansätzen problematisiert. Dementgegen wird die Aufgabe der Repräsentation nun in der Hervorbringung von Kollektiven gesehen: Indem sich Einzelne einem ,representative claim“ zuordnen, formieren sie ein Kollektiv (Saward 2006). Anstatt darin gegebene Kollektive abzubilden, liegt der Anspruch der Repräsentation darin, Identifikationsangebote zur Verfügung zu stellen, denen man sich anschließen kann oder eben nicht. So entstehen Kollektive, die allerdings immer nur temporär und partikular sind und jederzeit wieder auseinanderfallen können, sobald der claim nicht mehr angenommen wird. Darin liegt der proklamierte Konstruktivismus der Repräsentation (zum Stichwort ,constructivist turn“: Disch 2015; Disch et al. 2019).

Zweitens liegt in diesem neuen Bild des repräsentierten Kollektivs auch eine Betonung der temporalen Dimension von Repräsentation: Sowohl das Kollektiv als auch politische Formen erweisen sich als veränderlich. Repräsentation wird von ihrer institutionellen Form gelöst und stattdessen als kontinuierliches Wechselspiel von Konstituierung und Destituierung begriffen. Eine Demonstration auf der Straße kann dann ebenso repräsentativ sein wie eine NGO oder eine Partei im Parlament. Repräsentation wird zur fundamentalen Struktur politischen Handelns und verschwimmt dadurch mit ,,präsentischen“ Verständnissen der Demokratie (wie etwa Lorey 2020). Darin liegen die proklamierte Dynamisierung und Ubiquität der Repräsentation.

Während innerhalb der PT diese Erneuerungen eher als Produkte einer sich aktualisierenden theoretischen Diskussion angesehen werden (siehe Regina Kreides Beitrag in Niesen et al. 2020; Näsström 2015), wird in der IB der neue repräsentationstheoretische Diskurs als Reaktion auf Entwicklungen ,in der wirklichen Welt““ dargestellt. Referenzpunkte sind top-down formulierte Ansprüche, zivilgesellschaftliche Akteure stärker einbinden zu wollen, oder Proteste wie das sogenannte Battle of Seattle (1999), die zeigen, dass internationale Organisationen (IOs) als demokratisch illegitim wahrgenommen werden. Auch wenn die graduelle Etablierung eines Weltparlaments einmal als ein Pfad der internationalen Demokratisierung angesehen wurde (Falk und Strauss 2001), steht nun die Demokratisierung von IOs durch zivilgesellschaftliche Akteure im Mittelpunkt (Grigorescu 2015). Entgegen mancher Kritik aus der PT (Jörke 2019) mit Blick auf den Demokratieoptimismus in der 
IB, heißt dies nicht, dass IOs bereits als demokratisch angesehen werden (Holthaus 2018). Vielmehr geht man mit analogisierendem Verweis auf die Entwicklung nationalstaatlicher Repräsentation davon aus, dass wir uns in einer vordemokratischen (mit dem 18. Jh. vergleichbaren) Zeit befinden. Schließlich wählen die Vereinten Nationen noch die zivilgesellschaftlichen Akteure aus, mit denen sie arbeiten möchten (Goodin 2010). Transnationale Demokratie ist demzufolge eine emanzipatorische Zukunftsperspektive.

Innerhalb der IB entwickelt sich von diesen Diskussionen aus ein eigener Diskurs zur Frage, ob bzw. wie eine transnationale Zivilgesellschaft konzeptionell zu fassen ist (Bartelson 2006) und ob man die Repräsentationstheorie mit Blick auf zivilgesellschaftliche Akteure öffnen könnte und sollte. Diese Frage wird unterschiedlich beantwortet. Mit Referenz auf etablierte Theoretiker*innen wie Robert Dahl (1999) zweifeln einige, andere schlagen eine langsame Öffnung zur Erwägung der Repräsentation von Betroffenen vor (Näsström 2011), während wieder andere durch die Theorie der deliberativen Repräsentation eine radikale Öffnung und die Repräsentation von politischen Diskursen anregen, auch wenn dies, so die Kritik, Fragen nach politischer und epistemischer Ungleichheit marginalisiert (Dryzek et al. 2011). Schließlich werden zivilgesellschaftliche Akteure zum Teil auch mit einzelnen der ,alten“ noch nationalstaatlich gedachten Repräsentationstheorie entliehenen Begriffen wie Rechenschaftsplicht analysiert und normativ bewertet (Steffek und Ferretti 2009).

Etwas am Rande der Diskussion stehen feministische Beiträge, da sie sich mehr dem Problem von transnationalen In-und-Ausschlüssen widmen als der expliziten Weiterentwicklung oder Anwendung der Repräsentationstheorie. Dennoch sprechen sie direkt zu Fragen transnationaler Repräsentation. So erforscht beispielsweise Brooke Ackerly mit Methoden wie der teilnehmenden Beobachtung, wie im transnationalen Aktivismus Ausschlüsse zustande kommen, die es einigen privilegierten Gruppen des globalen Nordens erlauben, „für Frauen“ im Allgemeinen zu sprechen. Um diese Forschung zu betreiben, hat sich die Autorin bewusst und durch die ,,antisnownball method" bemüht, ihr eigenes von Privilegien gekennzeichnetes Milieu zu verlassen (Ackerly 2008).

Mit Blick auf das Potenzial vermehrter Verständigung zwischen PT und IB lassen sich drei Beobachtungen anstellen. Erstens hat es in der IB einen breiten und kritischen Diskurs zur sogenannten ,domestic analogy“ bzw. der (anti-)emanzipatorischen Orientierung an genuin westlichen nationalstaatlichen Repräsentationsformen gegeben (Little und McDonald 2013). Eine derartige Reflexion des eigenen Eurozentrismus sowie auch kritische Interventionen aus der postkolonialen Theorie heraus gibt es auch in der PT (etwa Koelble und Lipuma 2008; mit Blick auf Repräsentation natürlich auch Spivak 1988). Die Reflexion findet allerdings noch nicht systematisch Eingang in die genannten neuen Repräsentationstheorien. Zweitens nutzen einige Arbeiten die Repräsentationstheorie als Ressource zur kritischen Analyse von transnationaler Zivilgesellschaft (Holzscheiter 2016; Knappe 2020). Darin wird unter anderem gezeigt, dass westliche NGOs keineswegs die Interessen von nichtwestlichen Betroffenen an Wohlfahrtsleistungen repräsentieren, sondern deren Interessen in eigene Vorstellungen übersetzen (Koenig-Archibugi und Macdonald 2017). Dennoch muss - drittens - festgesellt werden, dass eine von der Reprä- 
sentationstheorie und/oder Feldforschung kritisch geleitete Analyse der zivilgesellschaftlichen Organisationen, welche die transnationale Demokratisierung antreiben sollen, die Ausnahme bleibt. Meist werden die eklatanten Unterschiede zwischen z. B. westlichen Gewerkschaften und diversen nichtwestlichen NGOs unzureichend thematisiert (Ottaway 2001).

Trotz der disziplinspezifischen Diskurse in der PT und IB zeigen Anschlüsse an Michael Sawards Konzeption der ,representative claims“ teilweise, wo der Dia$\log$ zwischen den Subdisziplinen schon gelingt. So analysiert beispielsweise Anna Holzscheiter mit Saward, die Rolle der Macht zu repräsentieren, die zivilgesellschaftlichen Kinderrechtsorganisationen des globalen Nordens vis-à-vis Kindergewerkschaften aus dem globalen Süden zukommt (Holzscheiter 2016). Dennoch wird das Potenzial des Dialogs nicht vollständig ausgeschöpft. Vor allem erachten wir es als wichtig - so wie wir noch in Bezug auf feministische Repräsentationstheorie zeigen werden - einen solchen Dialog nicht nur als Anwendung bestehender Theorien, sondern auch als kritische und empirisch informierte Arbeit an Theorien selbst zu begreifen.

Durch eine empirisch informierte, kritische Analyse ließe sich etwa darauf verweisen, dass Michael Sawards Verständnis von Repräsentation als einem performativen Akt, der Interessen und Identitäten der Repräsentierten erst im Vollzug der Repräsentation herstellt und gleichzeitig ausdehnbar ist auf informale Kontexte, durchaus demokratietheoretische Schwierigkeiten birgt: In der Idee eines freien Spiels von Repräsentationsansprüchen bleibt tendenziell unberücksichtigt, dass es strukturell unterlegene Positionen aufgrund ungleicher ökonomischer Ausgangsbedingungen, linguistischer Barrieren und systematischer Diskriminierung gibt. Der Vorschlag, dass sich unter jede Position und jedes Interesse ein Kollektiv versammeln könnte, bedeutet nicht, dass tatsächlich jedes dieser Anliegen gleichermaßen die Chance hat, gehört und anerkannt zu werden. In der Beschreibung von ,representative claims“ wird ein Markt von Repräsentationsansprüchen gezeichnet, in dem der im Wettbewerb erfolgreiche Repräsentationsanspruch eben der angemessene ist. Dabei gibt es, im Gegensatz zum formalisierten Wettbewerb der Parteien, der immerhin klar nach dem Prinzip der Rechtsgleichheit strukturiert ist, keine explizite Regulierung, durch die die unterschiedlichen Ausgangsbedingungen, aus denen heraus politische Subjekte aktiv werden, angeglichen werden könnten (mit dieser Kritik vergleiche Urbinati 2019, S. 75; Dovenney 2019; Martinez Mateo 2021). Dies ist gerade vor dem Hintergrund massiver globaler Ungleichheit von größter Relevanz. Denn wer sind am Ende die einbezogenen, repräsentierenden Akteure; welche Positionen finden Gehör und wer ist dazu in der Lage, selbst für sich zu sprechen (Dingwerth 2014, S. 1132)?

Gerade die Debatte um ,representative claims“ zeigt, wie sehr Forscher*innen bereits durch eine Öffnung zur Empirie PT weiterentwickeln (Montanaro 2018) sowie theoriegeleitet Analysen entwickeln, um empirische Repräsentationspraktiken auch normativ zu evaluieren (de Wilde 2020; Knappe und Schmidt 2021). Im angelsächsischen Kontext wird auch gern von ,angewandter politischer Theorie“ gesprochen (Theuns 2019). Feministische Repräsentationstheorie bzw. der Forschungsprozess, durch den sie entsteht, geht allerdings teilweise darüber hinaus. Der Forschungsprozess weist Affinitäten zu ethnografischer Forschung auf, denn in beiden Fällen 
setzen Forscher*innen Methoden wie die der teilnehmenden Beobachtung ein, um die (De-)Konstruktion von Repräsentationsansprüchen genau zu studieren. Auch sind ethnografische und feministische Forschung der Reflexion des eigenen Standpunkts und des Forschungsprozesses verpflichtet. Als eine eigene Besonderheit feministischer Forschung kann dabei gelten, dass es zum Teil eine Thematisierung und Aufwertung der emotionalen Dimension des Forschungsprozesses gibt (Åhäll 2018). Zudem kann, auch wenn gerade in den IB Feministinnen gezielt ihr eigenes, privilegiertes Milieu verlassen, feministische Forschung auch aus der Auseinandersetzung mit Erfahrungen und Unterdrückungsmechanismen im eigenen Feld entstehen, sowie aus der Reflektion über dessen transnationale Einbettung. Vor diesem Hintergrund ändert sich schließlich auch das Theorieverständnis. Der theoretische Anspruch liegt dann weniger in der Identifikation von Kriterien für ,gute“ transnationale Repräsentation (Wolkenstein und Wratil 2020). Ziel der Theorie ist vielmehr die Kritik an aufkommenden Repräsentationsverständnissen, Identitätskonstruktionen, wobei der Forschungsprozess, durch den diese Verunsicherungen entstehen, als „theoriegenerierend“ aufgewertet wird.

Von hier ausgehend, zeigt sich unserer Ansicht nach deutlich, wie gewinnbringend die Beschäftigung mit feministischen Repräsentationstheorien und -kritiken ist, nicht nur um das Nachdenken über Repräsentation zu verkomplizieren, sondern auch um das Verhältnis von politischer Theorie und Praxis als aufeinander verwiesen zu bestimmen.

\section{Feministische Interventionen}

Feministische Interventionen zu politischer Repräsentation zeigen auf, wie Theorie in Auseinandersetzung mit politischer Praxis und empirischen Arbeiten gewinnbringende Erkenntnisse zutage fördern kann. Im Speziellen geht es hier darum, unsichtbare Perspektiven in realweltlichen Zusammenhängen sichtbar zu machen:

[...] feminist, critical and decolonial scholars have argued that normative political theorizing reifies the perspectives of socially dominant groups, and is insufficiently attentive to the insights, experiences and interests of groups facing oppression [...] These and other criticisms have raised questions around whether normative political theory is sufficiently relevant to actual political problems and struggles against oppression (Ackerly et al. 2021, S. 2-3).

Wie also PT relevant sein kann in Bezug auf ,actual political problems“, das wollen wir anhand des Beitrags der feministischen Theorie zu einem erneuerten Repräsentationsverständnis verdeutlichen. Der Beitrag feministischer Theorie zum Verständnis von politischer Repräsentation liegt zunächst in einer Differenzierung des Verhältnisses von demokratischer Inklusion und Machtasymmetrien in und durch die Analyse realweltlicher Kämpfe um Repräsentation. Die feministische Theorie macht also darauf aufmerksam, dass demokratische Vorstellungen von Inklusion durchaus in Spannung stehen zu neueren konstruktivistischen Ansätzen der Repräsentationstheorie. So zeigen Feminist*innen wie auch postkoloniale Forscher*innen auf, inwiefern durch Repräsentation Exklusionsmechanismen wirken. In der femi- 
nistischen Tradition des Nachdenkens über Repräsentation geht es dabei nicht nur um die oben beschriebenen Fragen des Zugangs zu Repräsentation, sondern auch um das liberale Grundprinzip der Repräsentation, nach dem diese über Gruppenzugehörigkeiten hinweg operiert. Dieses Grundprinzip parlamentarischer Demokratie, dass jede*r Abgeordnete*r das gesamte Volk repräsentiert, steht nach feministischer Kritik folgender Realität gegenüber: Vor allem Männer repräsentieren Frauen, Akademiker*innen repräsentieren Arbeiter*innen und Arbeitslose, und weiße Deutsche repräsentieren People of Color. Dieser Umstand wird insofern problematisiert, als dass hier die zu repräsentierenden Gruppen durch den Akt der Repräsentation durch machtvollere Stellvertreter*innen unsichtbar gemacht und ausgeschlossen werden (Martinez Mateo 2019). In der historischen Analyse zeigt sich, wie die Vorstellung, dass Männer sehr gut auch Frauen repräsentieren können, zu der Schlussfolgerung führte, dass Frauen somit auch kein Wahlrecht benötigten (Cress 2018). Repräsentation hat also hier nicht den demokratischen Impetus einer Fürsprache und Inklusion, sondern wird - im Gegenteil - als machtvoller Akt der Ausschließung kritisiert: „Wurden lange Zeit Frauen als ,Andere“ konstruiert und aus der zu repräsentierenden Gruppe des Volkes exkludiert, weil sie ja von Männern repräsentiert würden, so schließt demokratische Repräsentation heute vor allem Migrant*innen als nicht zum fiktiven Volk gehörend aus und macht sie politisch nicht ,wahrnehmbar“" (Sauer 2011, S. 131).

Was wir in dieser empirisch rückgebundenen feministischen Kritik am konventionellen, am staatlichen Modell orientierten, Repräsentationsverständnis erkennen können, ist ein durchaus ambivalentes Verhältnis zu konstruktivistischen Ansätzen der Repräsentationstheorie. Obwohl beide Stränge die Kritik an einem statischen, essenzialisierten Repräsentationsverständnis eint, zeigt die feministische Kritik durch ihre Rückgebundenheit an realweltliche Ausgangslagen und politische Bewegungen auch die ,actual problems“ auf, die teilweise in Spannung stehen mit konstruktivistischen Ansätzen. Obwohl z. B. Birgit Sauer im oben genannten Zitat davon spricht, dass „Frauen“ als „Andere“ konstruiert werden im Akt der Repräsentation, also durchaus das konstruktivistische oder performative Moment von Repräsentation anerkannt wird, werden gleichsam die Grenzen von Repräsentation als einem kreativen Akt der Konstruktion aufgezeigt. So gibt es den starken Verweis hin zu einer ,realistischeren“ Auffassung von Repräsentation, in der es doch eine gewisse „Spiegelung“ von Repräsentierten und Repräsentant*innen gibt, nämlich durch Gruppenzugehörigkeiten. Hier wird nicht argumentiert, dass Repräsentant*innen die Interessen der Repräsentierten spiegeln, sondern dass Repräsentant*innen durch ihre zugeschriebenen und/oder angenommenen Identitäten Repräsentierte ,spiegeln“. Dies ist die Kernidee im Konzept von „,deskriptiver Repräsentation“ (Mansbridge 1999), das auf die Realität sozialer Ungleichheiten reagiert. Mansbridge definiert deskriptive Repräsentation wie folgt: ,,descriptive representatives ', that is, individuals who in their own backgrounds mirror some of the more frequent experiences and outward manifestations of belonging to the group“ (S. 628). Hier lenkt feministische Theorie zu deskriptiver Repräsentation den Blick darauf, dass die Formalisierung von Verfahren, z. B. durch Paritätsregeln, weniger günstige Ausgangsbedingungen von marginalisierten Personen ausgleichen kann und Repräsentation so transformativ wirken kann gegen Unterdrückung und Ungleichheit. Damit steht jedoch diese ,rea- 
listischere" Position in einer gewissen Spannung zu konstruktivistischen Ansätzen wie den der ,representative claims“ (Saward 2011).

Diese politiktheoretische Auseinandersetzung mit dem transformativen Potenzial deskriptiver Repräsentation beschränkt sich nicht nur auf Paritätsregeln in politischen Institutionen, sondern nimmt gleichwohl Debatten und Strömungen jenseits institutionalisierter Politik auf. Die Frage, wie Darstellung (ästhetische Repräsentation) sich verschränkt mit politischer Repräsentation ist für die feministische Repräsentationskritik zentral (Disch 2016). Wie etwas oder jemand dargestellt wird, kann für politische Ziele genutzt werden. So steht beispielsweise die Film- und Fernsehbranche immer wieder in der Kritik, wenig bis keine Frauen, People of Color oder Menschen mit Behinderung in ihren Filmproduktionen, Talkshows oder Nachrichtensendungen zu zeigen. „Representation matters“ ist gerade auch in den letzten Jahren wieder ein prominenter Slogan geworden, über die Grenzen der Filmbranche hinweg. Gerade zu dieser Frage sind wichtige Ansätze aus der postkolonialen Theorie zu nennen (vergleiche Gutiérrez Rodríguez 2008; Steyerl und Gutiérrez Rodríguez 2012).

Dieser feministische Ansatz steht natürlich im Gegensatz zur konstruktivistischen Repräsentationstheorie, indem sich auch repräsentierte Gruppen erst durch den Akt der Repräsentation konstituieren und somit nicht zu spiegeln sind. Während also feministische Interventionen immer wieder darauf angewiesen sind, bestimmte Identitäten als gegeben anzunehmen, um ihnen Gehör zu verschaffen, werden in der theoretischen (konstruktivistischen) Debatte das Aufrufen fester Identitäten und Interessen (zu Recht) in Zweifel gezogen und machtkritisch hinterfragt. In diesem produktiven Spannungsfeld zwischen politischer Praxis und Theorie (Disch 2016) eröffnet sich das Dilemma zwischen konstruktivistischer Repräsentationskritik und transformativem Repräsentationsanspruch: „While feminist activists worked from the commonsense premise that the objectivation of women in mass culture fostered discrimination, even violence, [...] some feminist theorists develop the counterintuitive argument that representations of women do not refer to or stand for real women“" (Disch 2016, S. 782).

So muss, ausgehend von der konstruktivistischen Perspektive, auch auf die Schwierigkeiten eines solchen Verständnisses von Repräsentation als Sichtbarmachung einzelner (durch Identitätsmerkmale definierter) Gruppenmitglieder hingewiesen werden (Schaffer 2008). Schließlich ist es gerade auch die feministische Theorie, die diesen realistischen Ansatz von Repräsentation kritisiert und Repräsentation konstruktivistisch versteht.

Um auf diese Kritik zu reagieren, ist es also wichtig, den Konstruktivismus der neueren Repräsentationsansätze nicht aufzugeben. Anstatt - wie in deskriptiver Repräsentation - von gegebenen und identifizierbaren Kollektiven auszugehen, kann es produktiv sein, die Performativität von Repräsentation anzuerkennen und zu affirmieren. Es liegt ein emanzipatorisches Moment darin, dass politische Kollektive und Identitäten erst durch Repräsentation hervorgebracht werden (und dabei auch unfertig und veränderbar bleiben). Gerade aus feministischen Debatten lässt sich hierfür viel lernen, denn das darin aufgerufene Kollektivsubjekt „Frau“ ist freilich immer schon umstritten - und etwa von schwarzen Frauen kritisiert worden, die sich in dieser Bestimmung nicht mitgemeint sahen (etwa: Carby 1997; Crenshaw 1989). 
Was ist zu lernen aus dem Dialog von Praxis und Theorie, den feministischen Forscher*innen hier aufnehmen und mit dem sie Theorie weiterentwickeln? Um sich demokratischen Repräsentationsformen anzunähern, helfen Idealtypen demokratischer Institutionen nicht unbedingt weiter. Vielmehr sind es vielleicht die, imperfect yet meaningful democratic practices“ (Little und Macdonald 2013, S. 789), die es zu untersuchen gilt.

\section{Schluss}

Wie wir dargelegt haben, zeigt sich, dass in der feministischen Perspektive eine entscheidende Ressource liegt, um zu verstehen, wie normative Theorie an empirische Gesellschaftsanalyse rückgebunden werden kann und wie, ausgehend von der kritischen Analyse, Theorieansätze und Begriffe neu bewertet und weiterentwickelt werden können. Die feministische Perspektive leistet eine doppelte Verschränkung von Theorie und Empirie, die sowohl rein normative Theorieansätze als auch eine theorieferne, empirische Analysen, die Idee von naturwissenschaftlicher Objektivität verpflichteten bleiben, ergänzt. Schließlich definiert sich die feministische Perspektive, einerseits, durch den Gebrauch ethnografischer Methoden und der Reflexion der Affekte, die von Forschung zu transnationaler Repräsentation oder Einbettung von Repräsentationspraktiken in transnationale Machtasymmetrien, ausgelöst werden. Andererseits kennzeichnet sich die feministische Perspektive durch das stetige Infragestellen von theoretischen Versprechen, wie es auch in Diskursen zu deskriptiver Repräsentation zu erkennen ist.

Zusätzlich zur Herausarbeitung von feministischen Erkennungsmerkmalen haben wir konkret feministische Ansätze und Interventionen zur Repräsentationstheorie und -praxis diskutiert, an denen sich die Besonderheit der feministischen Perspektive in eigener Weise zeigt. Wie ausgeführt, sind zunächst wichtige theoretische Innovationen zu traditionellen staatszentrierten Repräsentationsansätzen in der PT zu verzeichnen, die einerseits auf den Konstruktivismus der Repräsentation verweisen und andererseits eine Dynamisierung der Repräsentationsbeziehung beanspruchen. Diese theoretische Innovation ist zugleich von einer empirischen Verschiebung realer Repräsentationsverhältnisse durch deren Verlagerung auf den transnationalen Raum angetrieben sowie von den Problemen und Fragen, die sich aus diesen Verschiebungen ergeben. Deutlich wird dabei allerdings, dass durch bestehende disziplinäre Engführungen bestimmte Problematiken, die auch in dieser theoretischen Neubestimmung von Repräsentation liegen, nicht zu Genüge beachtet werden. Hier setzen die feministischen Interventionen in die Repräsentationstheorie und -Forschung an, die einerseits gegen den neuen Konstruktivismus der Repräsentation darauf verweisen, dass die Spielräume zur Teilhabe an einer konstruktivistisch gedachten Repräsentationsbeziehung durch ungleiche Ausgangsbedingungen strukturiert sind, die sich entlang von Identitätszuschreibungen abspielen, sodass der Konstruktivismus hier seine Grenzen hat. Gegen die Dynamisierung und Ent-Formalisierung der Repräsentation wenden feministische Ansätze andererseits ein, dass darin eine Verfestigung sozialer Ungleichheiten droht, der durch spezifische und gezielte Formalisierungen entgegengewirkt werden kann - etwa in der Einführung von Quoten. So zeigt sich, 
dass es stets der kritische Blick auf die soziale Realität ist, der letztlich auch zu einer Revision theoretischer Ansätze beiträgt. Die feministische Perspektive greift kritisch Diagnosen globaler Demokratie als auch theoretische Neubestimmungen der Repräsentation auf. Dazu aber muss feministische Theorie und Forschung sowohl in der IB als auch in der PT in viel stärkerem Maße als bisher der Fall als ein zentraler und eigenständiger Beitrag in den jeweiligen (Teil-)Disziplinen anerkannt werden, von dem sich - theoretisch, empirisch wie methodisch - vieles lernen lässt, was anderswo zu kurz kommt.

Von feministischen Ressourcen können sowohl die PT als auch die IB und disziplinübergreifende Dialoge profitieren - gerade dort, wo Diskrepanzen zwischen normativer Theorie und empirischer Forschung beklagt und nach Wegen gesucht wird, beides stärker miteinander zu verknüpfen. Dies ist nicht nur, wie bereits ausgeführt, in der Debatte (Niesen et al. 2020) der Fall, der für die vorliegende Diskussion den Ausgangspunkt bildete. Diskrepanzen zwischen normativer Theorie und empirischer Analyse stellen darüber hinaus und auch jenseits des deutschen Kontexts ein vielerorts diagnostiziertes Problem dar (vgl. etwa Herzog und Zackas 2019; sowie Snidal und Reus-Smit 2008, S. 7). Würde in solchen Kontexten der Beitrag und die Tragweite feministischer Ansätze und Methodologien vollständig erkannt, könnte bei der Verknüpfungsforderung klarerweise auf bereits bestehende Arbeit zurückgegriffen werden.

Danksagung Wir möchten Anna Holzscheiter sowie den Gutacher*innen und Herausgeber*innen für wertvolle Kommentare und Fiona Ganzer für hervorragende Arbeit zu diesem Artikel danken.

Funding Open Access funding enabled and organized by Projekt DEAL.

Open Access Dieser Artikel wird unter der Creative Commons Namensnennung 4.0 International Lizenz veröffentlicht, welche die Nutzung, Vervielfältigung, Bearbeitung, Verbreitung und Wiedergabe in jeglichem Medium und Format erlaubt, sofern Sie den/die ursprünglichen Autor(en) und die Quelle ordnungsgemäß nennen, einen Link zur Creative Commons Lizenz beifügen und angeben, ob Änderungen vorgenommen wurden.

Die in diesem Artikel enthaltenen Bilder und sonstiges Drittmaterial unterliegen ebenfalls der genannten Creative Commons Lizenz, sofern sich aus der Abbildungslegende nichts anderes ergibt. Sofern das betreffende Material nicht unter der genannten Creative Commons Lizenz steht und die betreffende Handlung nicht nach gesetzlichen Vorschriften erlaubt ist, ist für die oben aufgeführten Weiterverwendungen des Materials die Einwilligung des jeweiligen Rechteinhabers einzuholen.

Weitere Details zur Lizenz entnehmen Sie bitte der Lizenzinformation auf http://creativecommons.org/ licenses/by/4.0/deed.de.

Interessenkonflikt L. Holthaus, H. Knappe und M. Martinez Mateo geben an, dass kein Interessenkonflikt besteht.

\section{Literatur}

Åhäll, Linda. 2018. Affect as methodology: feminism and the politics of emotion. International Political Sociology 12(1):36-52. https://doi.org/10.1093/ips/olx024.

Ackerly, Brooke. 2008. Feminist methodological reflection. In Qualitative methods in international relations, Hrsg. Audie Klotz, Deepa Prakash, 28-42. London: Palgrave Macmillan. https://doi.org/10. 1057/9780230584129_3. 
Ackerly, Brooke, Luis Cabrera, Fonna Forman, Genevieve F. Johnson, Chris Tenove, und Antje Wiener. 2021. Unearthing grounded normative theory: practices and commitments of empirical research in political theory. Critical Review of International Social and Political Philosophy 38(3):1-27. https:// doi.org/10.1080/13698230.2021.1894020.

Bartelson, Jens. 2006. Making sense of global civil society. European Journal of International Relations 12:371-395. https://doi.org/10.1177/2F1354066106067348.

Carby, Hazel V. 1997. White woman listen! Black feminism and the boundaries of sisterhood. In Materialist feminism. A reader in class, difference and women's lives, Hrsg. Rosemary Hennessy, Ingraham Chrys, 111-128. New York: Routledge.

Crenshaw, Kimberle. 1989. Demarginalizing the intersection of race and sex: a black feminist critique of antidiscrimination doctrine, feminist theory and antiracist politics. University of Chicago Legal Forum 1:139-167.

Cress, Anne. 2018. Feministische Repräsentationskritik: (Dis-)Kontinuitäten von den ersten deutschen Frauenbewegungen bis in die Gegenwart. FEMINA POLITICA - Zeitschrift für feministische Politikwissenschaft 27:25-39. https://doi.org/10.3224/feminapolitica.v27i2.03.

Dahl, Robert A. 1999. Can international organizations be democratic? A skeptic's view. In Democracy's edges, Hrsg. Ian Shapiro, Casiano Hacker-Cordon, 19-36. Cambridge: Cambridge University Press.

De Wilde, Pieter. 2020. The quality of representative claims: uncovering a weakness in the defense of the liberal world order. Political Studies 68:271-292. https://doi.org/10.1177/2F0032321719845199.

Deitelhoff, Nicole, und Christopher Daase. 2015. Herrschaftszeiten: Internationale Politische Theorie als Gesellschaftstheorie der internationalen Beziehungen. Zeitschrift für Politische Theorie 6:141-158. https://doi.org/10.3224/zpth.v6i2.22874.

Dingwerth, Klaus. 2014. Global democracy and the democratic minimum: why a procedural account alone is insufficient. European Journal of International Relations 20:1124-1147. https://doi.org/10.1177/ 2F1354066113509116.

Disch, Lisa. 2015. The 'constructivist turn' in democratic representation: a normative dead-end? Constellations 22:487-499. https://doi.org/10.1111/1467-8675.12201.

Disch, Lisa. 2016. Representation. In The Oxford handbook of feminist theory, Hrsg. Lisa Disch, Mary Hawkesworth, 781-802. New York: Oxford University Press.

Disch, Lisa, Mathijs van de Sande, und Nadia Urbinati. 2019. The constructivist turn in political representation. Edinburgh: Edinburgh University Press.

Dovenney, Mark. 2019. The improper politics of representation. In The constructivist turn in political representation, Hrsg. Lisa Disch, Mathijs van de Sande, und Nadia Urbinati, 224-238. Edinburgh: Edinburgh University Press.

Dovi, Suzanne. 2006. Political representation. In The Stanford encyclopedia of philosophy, Hrsg. Edward N. Zalta https://plato.stanford.edu/entries/political-representation/.

Dryzek, John S., André Bächtiger, und Karolina Milewicz. 2011. Toward a deliberative global citizen assembly. Global Policy 2:33-42. https://doi.org/10.1111/j.1758-5899.2010.00052.x.

Duso, Giuseppe. 2006. Die moderne politische Repräsentation: Entstehung und Krise des Begriffs. Berlin: Duncker \& Humblot.

Falk, Richard, und Andrew Strauss. 2001. Toward global parliament. Foreign Affairs 80:212-220. https:// doi.org/10.2307/20050054.

Goodin, Robert E. 2010. Global democracy: in the beginning. International Theory 2:175-209. https://doi. org/10.1017/S1752971910000060.

Grigorescu, Alexandru. 2015. Democratic intergovernmental organisations? Normative pressures and decision-making rules. Cambridge: Cambridge University Press.

Gutiérrez Rodríguez, Encarnación. 2008. Postkolonialismus: Subjektivität, Rassismus und Geschlecht. In Handbuch Frauen- und Geschlechterforschung: Theorie, Methoden, Empirie, Hrsg. Ruth Becker, Beate Kortendiek, 267-275. Wiesbaden: VS.

Herzog, Lisa, und Bernardo Zackas. 2019. Fieldwork in political theory: five arguments for an ethnographic sensibility. British Journal of Political Science 49(2):763-784. https://doi.org/10.1017/ S0007123416000703.

Hofmann, Hasso. 2003. Repräsentation: Studien zur Wort- und Begriffsgeschichte von der Antike bis ins 19. Jahrhundert. Berlin: Duncker \& Humblot.

Holthaus, Leonie. 2018. Pluralist democracy in international relations. L.T. Hobhouse, G.D.H. Cole and David Mitrany. New York: Palgrave.

Holzscheiter, Anna. 2016. Representation as power and performative practice: global civil society advocacy for working children. Review of International Studies 42:205-226. https://doi.org/10.1017/ S0260210515000145. 
Jörke, Dirk. 2019. Die Größe der Demokratie. Über die räumliche Dimension von Herrschaft und Partizipation. Frankfurt: Suhrkamp.

Knappe, Henrike. 2020. Representation as practice: agency and relationality in transnational civil society. Journal of International Relations and Development https://doi.org/10.1057/s41268-020-00197-6.

Knappe, Henrike, und Oscar Schmidt. 2021. Making representations: the SDG process and major groups' images of the future. Global Environmental Politics 21(2):23-43. https://doi.org/10.1162/glep_a_ 00599 .

Koelble, Thomas A., und Edward Lipuma. 2008. Democratizing democracy: a postcolonial critique of conventional approaches to the 'measurement of democracy'. Democratization 15:1-28. https://doi. org/10.1080/13510340701768075.

Koenig-Archibugi, Matthias, und Kate Macdonald. 2017. The role of beneficiaries in transnational regulatory processes. The ANNALS of the American Academy of Political and Social Science 670:36-57. https://doi.org/10.1177/2F0002716216688862.

Little, Adrian, und Kate Macdonald. 2013. Pathways to global democracy? Escaping the statist imaginary. Review of International Studies 39:789-813. https://doi.org/10.1017/S0260210512000551.

Lorey, Isabell. 2020. Demokratie im Präsens. Eine Theorie der politischen Gegenwart. Berlin: Suhrkamp.

Macdonald, Terry. 2008. Global stakeholder democracy. Power and representation beyond liberal states. Oxford: Oxford University Press.

Mansbridge, Jane. 1999. Should blacks represent blacks and women represent women? A contingent 'yes'. The Journal of Politics 61(3):628-657. https://doi.org/10.2307/2647821.

Martinez Mateo, Marina. 2018. Politik der Repräsentation. Zwischen Formierung und Abbildung. Wiesbaden: Springer.

Martinez Mateo, Marina. 2019. Füreinander Sprechen: Zu einer feministischen Theorie der Repräsentation. Leviathan 47:331-353. https://doi.org/10.5771/0340-0425-2019-3-331.

Martinez Mateo, Marina. 2021. Das Problem des Volkes: Möglichkeiten einer Kritik der Repräsentation. In Im Namen des Volkes: Zur Kritik politischer Repräsentation, Hrsg. Marvin Neubauer, Max Stange, Charlott Resske, und Frederik Doktor, 177-200. Tübingen: Mohr Siebeck.

Montanaro, Laura. 2018. Who elected Oxfam? A democratic defense of self-appointed representatives. Cambridge: Cambridge University Press.

Näsström, Sofia. 2011. The challenge of the all-affected principle. Political Studies 59:116-134. https:// doi.org/10.1111/j.1467-9248.2010.00845.x.

Näsström, Sofia. 2015. Democratic representation beyond election. Constellations 22:1-12. https://doi.org/ $10.1111 / 1467-8675.12123$.

Niesen, Peter. 2010. Internationale Politische Theorie: Eine disziplingeschichtliche Einordnung. Zeitschrift für Internationale Beziehungen 17:267-277. https://doi.org/10.5771/0946-7165-2010-2-267.

Niesen, Peter, Armin Schäfer, Michael Zürn, Wolfgang Knöbl, Claudia Landwehr, Regina Kreide, und Eva Marlene Hausstainer. 2020. Politische Theorie und Ideengeschichte im Wissenschaftssystem. Politische Vierteljahresschrift 61:747-769. https://doi.org/10.1007/s11615-020-00276-w.

Ottaway, Marina. 2001. Corporatism goes global: international organizations, nongovernmental organization networks, and transnational business. Global Governance 7:265-292.

Pitkin, Hanna F. 1972. The concept of representation. Berkeley/Los Angeles: University of California Press.

Sauer, Birgit. 2011. ,Only paradoxes to offer'? Feministische Demokratie- und Repräsentationstheorie in der ,Postdemokratie‘. Österreichische Zeitschrift für Politikwissenschaft 40(2):125-138. https://doi. org/10.15203/ozp.567.vol40iss2.

Saward, Michael. 2006. The representative claim. Oxford: Oxford University Press.

Saward, Michael. 2011. Slow theory: taking time over transnational democratic representation. Ethics \& Global Politics 4(1):1-18. https://doi.org/10.3402/egp.v4i1.6416.

Schaffer, Johanna. 2008. Ambivalenzen der Sichtbarkeit: Über die visuellen Strukturen der Anerkennung. Bielefeld: transcript.

Schmitter, Philippe C. 2009. Re-presenting representation. Government and Opposition 44:476-490. https://doi.org/10.1111/j.1477-7053.2009.01300.x.

Snidal, Duncan, und Christian Reus-Smit. 2008. Between utopia and reality: the practical discourses of international relations. In The Oxford handbook of international relations, Hrsg. Cristian Reus-Smit, 3-39. Oxford: Oxford University Press.

Spivak, Gayatri C. 1988. Can the subaltern speak? In Marxism and the interpretation of culture, Hrsg. Cary Nelson, Larry Grossberg, 271-313. Basingstoke: Macmillan Education. 
Steffek, Jens, und Maria Paola Ferretti. 2009. Accountability or 'good decisions'? The competing goals of civil society participation in international governance. Global Society 23:37-57. https://doi.org/10. 1080/13600820802556736.

Steyerl, Hito, und Encarnación Gutiérrez Rodríguez. 2012. Spricht die Subalterne deutsch? Migration und postkoloniale Kritik. Münster: Unrast.

Theuns, Tom. 2019. The legitimacy of free trade agreements as tools of EU democracy promotion. Cambridge Review of International Affairs 32:3-21. https://doi.org/10.1080/09557571.2018.1552247.

Urbinati, Nadia. 2019. Judgment alone: cloven citizenship in the era of the Internet. In Creating political presence: the new politics of democratic representation, Hrsg. Dario Castiglione, Johannes Pollak, 61-85. Chicago: University of Chicago Press.

Urbinati, Nadia, und Mark E. Warren. 2008. The concept of representation in contemporary democratic theory. Annual Review of Political Science 11:387-412. https://doi.org/10.1146/annurev.polisci.11. 053006.190533.

Wolkenstein, Fabio, und Christopher Wratil. 2020. Multidimensional representation. American Journal of Political Science 65(4):862-876. https://doi.org/10.1111/ajps.12563. 